\title{
An uncommon cause of hemoptysis in pulmonary tuberculosis
}

\section{Nitesh Gupta, Juvva Kishan Srikanth, Puneet Garg, Pranav Ish, Shibdas Chakrabarti}

VMMC and Safdarjung Hospital, New Delhi, India, New Delhi, India

A sixty-year-old woman, diabetic on oral hypoglycemic, presented with complaints of low-grade fever, cough with scanty sputum, breathlessness since 3 weeks followed by streaky hemoptysis for five days. The CT thorax showed a right middle lobe collapse with bilateral diffuse random nodules. On bronchoscopic examination, broncho-alveolar lavage (BAL) was collected from the right upper lobe. The right middle lobe opening was narrowed and was bleeding on touch, and the patient lost around $700 \mathrm{ml}$ of blood. The bronchoscope was wedged in the bleeding secondary bronchus to tamponade it, the patient was placed in dependent position and hemostasis was achieved. BAL sent for cartridge based nucleic acid amplification test (CBNAAT) was positive for mycobacterium tuberculosis complex, rifampicin sensitive. Subsequently, a CT pulmonary angiography (CTPA) was done (Figure 1).
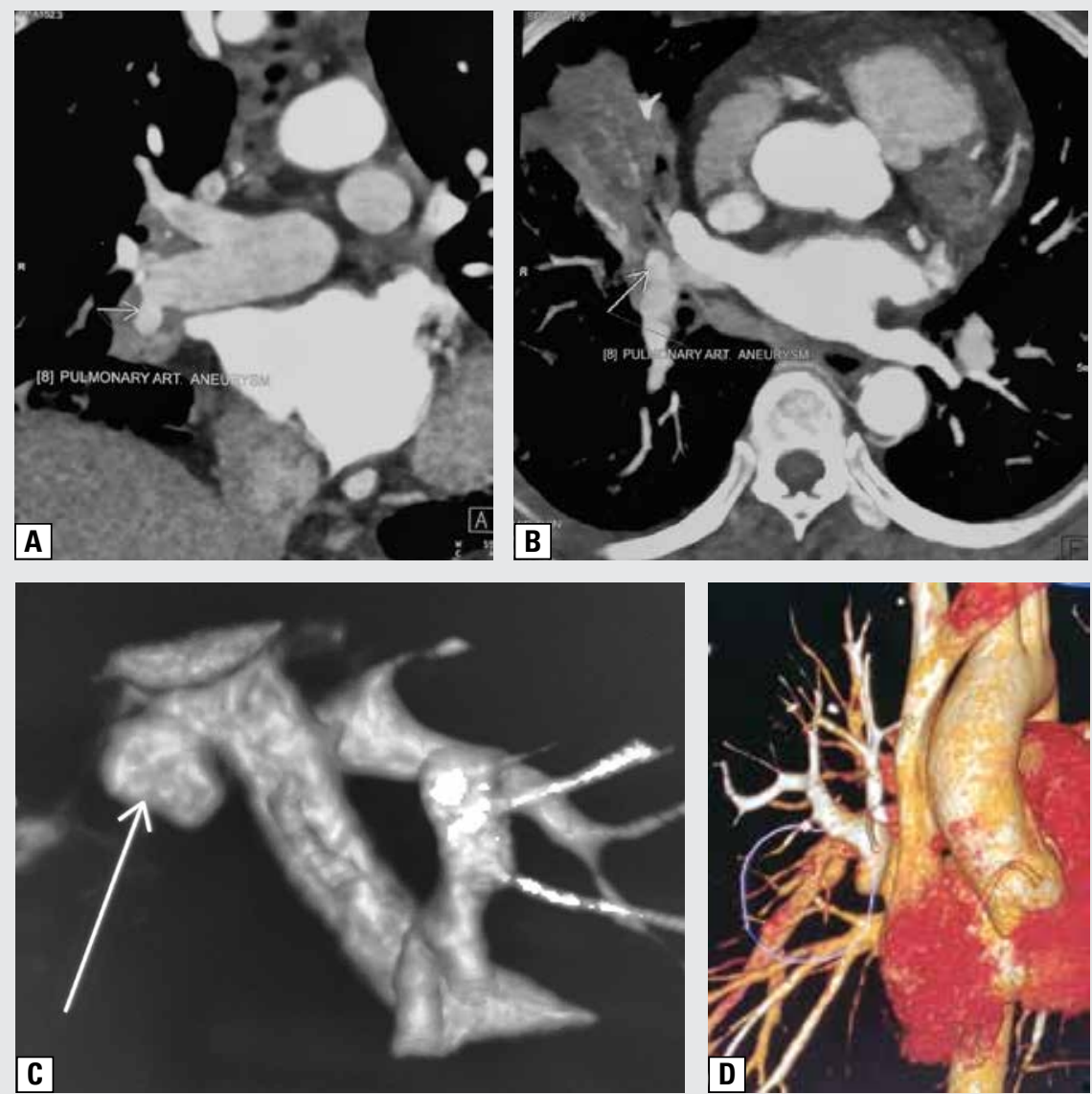

Figure 1. A. Coronal CT image showing a wide neck aneurysm arising from the proximal right descending pulmonary artery; B. Axial CT image showing a wide neck aneurysm from the right descending pulmonary artery with associated collapse of the right middle lobe; C. Volumerendered CT image showing aneurysm from the pulmonary artery; D. Volume-rendered CT image showing aneurysm arising from the right descending pulmonary artery and facing anteriorly

Address for correspondence: Pranav Ish, Department of Pulmonary, Critical Care And Sleep Medicine, Vmmc and Safdarjung Hospital, New Delhi, India; e-mail: pranavish2512@gmail.com

DOI: 10.5603/ARM.2019.0067

Received: 13.08 .2019

Copyright (C) 2019 PTChP

ISSN 2451-4934

Conflict of interest: none declared 
CTPA revealed a pseudoaneurysm of the right main pulmonary artery causing narrowing of the middle lobe bronchus. Thus, the final diagnosis of pulmonary tuberculosis with pseudoaneurysm of the right main pulmonary artery causing right middle lobe collapse and massive hemoptysis was made. The patient was started on anti-tubercular therapy, subsequently symptoms of fever, breathlessness and hemoptysis resolved within 2 weeks.

The common causes of hemoptysis in pulmonary tuberculosis include endobronchial tuberculosis, tubercular cavity, bronchiectasis and rarely vascular aneurysms. Also, the sequels and complications to be considered are apergilloma, scar carcinoma; airway lesions such as bronchiectasis and broncholithiasis; or vascular lesions such as pulmonary or bronchial arteritis and thrombosis or dilatation. Rasmussen's aneurysm is another cause, but it is found adjacent to a tubercular cavity, commonly involving the upper lobe [1]. However, the present case had a main pulmonary artery pseudoaneurysm causing compression collapse of the right middle lobe. In this post-antibiotic era, both tuberculosis and syphilis are rare causes of aneurysms; vasculitis and malignancies are now the most common etiologies [2].

In review of the literature, aortic aneurysms occurred more frequently in tuberculosis, and mostly resulted in deaths [3]. A single case report of a main pulmonary artery pseudoaneurysm in tubercular empyema also described a massive hemoptysis leading to death [4].

The gold standard for diagnosis is pulmonary angiography, but this is now largely superseded by noninvasive CT angiography and 3-D reconstruction. On CECT, a pseudoaneurysm appears as an enhancing mass adjacent to a vessel, which is isodense to the central pulmonary arteries.

Treatment of such pseudoaneurysms remains controversial. Several surgical techniques such as aneurysmorrhaphy, lobectomy, bi-lobectomy, aneurysmectomy and pneumonectomy have been tried with variable results. Surgery carries a high risk, especially if there is severe pulmonary artery hypertension. So, endovascular treatment can be offered as initial therapy. However, there are no consensus guidelines, and in non-urgent asymptomatic patients, conservative management can be considered, especially in treatable underlying etiologies [5].

To conclude, massive hemoptysis in pulmonary tuberculosis can have varied etiologies. A high index of suspicion must be kept for aneurysmal bleeding as it is rare, but a potentially life-threatening cause.

\section{References:}

1. Plessinger VA, Jolly PN. Rasmussen's aneurysms and fatal hemorrhage in pulmonary tuberculosis. Am Rev Tuberc. 1949; 60(5): 589-603, illust, doi: 10.1164/art.1949.60.5.589, indexed in Pubmed: 15392763.

2. Chen Y, Gilman MD, Humphrey KL, et al. Pulmonary artery pseudoaneurysms: clinical features and CT findings. AJR Am J Roentgenol. 2017; 208(1): 84-91, doi: 10.2214/AJR.16.16312, indexed in Pubmed: 27656954.

3. Xue J, Yao Y, Liu L. Treatment of tuberculous aortic pseudoaneurysm associated with vertebral tuberculosis: A case series and a literature review. Medicine (Baltimore). 2018; 97(15): e0382, doi: 10.1097/MD.0000000000010382, indexed in Pubmed: 29642195.

4. Verma A, Prakash V, Kant S, et al. Pulmonary artery pseudoaneurysm: An uncommon fatal complication associated with tubercular empyema. Journal of Mahatma Gandhi Institute of Medical Sciences. 2015; 20(2): 176, doi: 10.4103/0971-9903.164249.

5. Park HS, Chamarthy MR, Lamus D, et al. Pulmonary artery aneurysms: diagnosis \& endovascular therapy. Cardiovasc Diagn Ther. 2018; 8(3): 350-361, doi: 10.21037/cdt.2018.04.01, indexed in Pubmed: 30057881. 